\title{
Erratum to: Towards Higgs boson production in gluon fusion to NNLO in the MSSM
}

\author{
Alexey Pak, Matthias Steinhauser ${ }^{\mathrm{a}}$, Nikolai Zerf \\ Institut für Theoretische Teilchenphysik, Karlsruhe Institute of Technology (KIT), 76128 Karlsruhe, Germany
}

Received: 9 August 2012 / Revised: 9 September 2012 / Published online: 25 October 2012

(c) Springer-Verlag Berlin Heidelberg and Società Italiana di Fisica 2012

\section{Erratum to: Eur. Phys. J. C (2011) 71:1602} DOI 10.1140/epjc/s10052-011-1602-9

There are several errors in the three-loop coefficient of order $\left(\alpha_{s}^{(5)}\right)^{3} x_{t s}^{2}$ in Eq. (18). The corrected result reads

$$
\begin{aligned}
C_{1}^{\overline{\mathrm{DR}}}= & -\frac{\alpha_{s}^{(5)}}{3 \pi} \frac{c_{\alpha}}{s_{\beta}}\left\{\cdots+\left(\frac{\alpha_{s}^{(5)}}{\pi}\right)^{2}[\ldots\right. \\
& +\left(\frac{30779857}{648000}-\frac{6029}{10800} l_{\text {susy }}-\frac{475}{288} l_{\text {susy }}^{2}\right. \\
& -\frac{20407}{10800} l_{\mathrm{t}}+\frac{26}{45} l_{\text {susy }} l_{\mathrm{t}}-\frac{377}{1440} l_{\mathrm{t}}^{2}-\frac{15971}{576} \zeta_{3} \\
& +\left(-\frac{3910697}{216000}+\frac{63}{4} \zeta_{3}+\frac{3307}{4800} l_{\text {susy }}+\frac{131}{576} l_{\text {susy }}^{2}\right. \\
& \left.\left.\left.\left.+\frac{5113}{14400} l_{\mathrm{t}}-\frac{101}{1440} l_{\text {susy }} l_{\mathrm{t}}+\frac{3}{320} l_{\mathrm{t}}^{2}\right) n_{l}\right) x_{t s}^{2}\right]\right\} \\
& +\mathcal{O}\left(x_{t s}^{4}\right)+\mathcal{O}\left(\frac{\mu_{\text {susy }}}{m_{\text {susy }}} x_{t s}^{2}\right) .
\end{aligned}
$$

The change in the numerical results is negligible.

The relation between $C_{g}$ and $C_{1}$ in Eq. (4) has to be changed to $C_{g}=C_{1} / C_{1}^{(0)} / B^{(5)}$ where $C_{1}^{(0)}$ is the coefficient of $\alpha_{s}^{(5)}$ in $C_{1}$.

In Eq. (20) " $\left[1+\left(1-\tau_{t}\right)\right] f\left(\tau_{t}\right)$ " has to be replaced by "[1+(1- $\left.\left.\tau_{t}\right) f\left(\tau_{t}\right)\right]$ ".

In Eq. (22) the "1" on the r.h.s. has to be replaced by “ $\Sigma^{(0)} ":$

$\Sigma=\Sigma^{(0)}+\frac{\alpha_{s}^{(5)}\left(\mu_{s}\right)}{\pi} \Sigma^{(1)}+\left(\frac{\alpha_{s}^{(5)}\left(\mu_{s}\right)}{\pi}\right)^{2} \Sigma^{(2)}+\cdots$,

In Eq. (23) " $\sigma$ LO" has to be replaced by “ $\sigma_{0}$ ".

The online version of the original article can be found under doi:10.1140/epjc/s10052-011-1602-9.

a e-mail: matthias.steinhauser@uka.de 